\title{
Normal Values of Echocardiographic Measurements. A Population-Based Study
}

\author{
Paulo Roberto Schvartman, Flávio Danni Fuchs, Alex Gules Mello, Maikel Coli, Mario Schvartman, \\ Leila Beltrami Moreira
}

Porto Alegre, RS - Brazil

\begin{abstract}
Objective - To describe echocardiographic measurements and left ventricular mass in a population sample of healthy adults inhabitants of the urban region of Porto Alegre.

Methods - An analytical, observational, populationbased, cross-sectional study was done. Through a multistage probability sample, 114 individuals were selected to be submitted to a M-mode and two-dimensional echocardiogram with color Doppler. The analyses were restricted to healthy participants. Echocardiographic measurements were described by mean, standard deviation, 95 percentile and $95 \%$ confidence limits.
\end{abstract}

Results - A total of 100 healthy participants, with several characteristics similar to those from the original population, had a complete and reliable echocardiographic examination. The measurements of aorta, left atrium, interventricular septum, left ventricle in systole and diastole, left posterior wall and left ventricular mass, adjusted or not for body surface area or height, were significantly higher in males. The right ventricle size was similar among the genders. Several echocardiographic measurements were within standard normal limits. Interventricular septum, left posterior wall and left ventricular mass, adjusted or not for anthropometric measurements, and aortic dimensions had lower mean and range than the reference limits.

Conclusion - The means and estimates of distribution for the measurements of interventricular septum, left posterior wall and left ventricular mass found in this survey were lower than those indicated by the international literature and accepted as normal limits.

Key words: echocardiogram, normal values

Hospital de Clínicas de Porto Alegre da Universidade Federal do Rio Grande do Sul Mailing address: Flávio Danni Fuchs - Serviço de Cardiologia - Hospital de Clínicas de Porto Alegre - Rua Ramiro Barcelos, 2350 - 90035-003 - Porto Alegre, RS - Brazil - E-mail: ffuchs@ @cpa.ufrgs.br
The normal values for echocardiographic measurements presented in textbooks ${ }^{1}$ are heterogeneous and at times inconsistent. They are based in original studies of individuals who were evaluated for suspected heart disease, which was not confirmed thereafter ${ }^{2,3 .}$. These individuals may be free of detectable cardiovascular disease determined by echocardiography; however, they do not represent the normal population. Other studies do not indicate the origin of the sample or use family members of the patients or employees of the hospital to determine normal values ${ }^{4}$.

The normal echocardiographic parameters of a population should come from a random sample of the population, not hospitalized and asymptomatic. No such study has been done in Brazil using representative samples of adults free of cardiovascular diseases. In the international literature, studies do exist of normal values of the dimensions, function, and left ventricular mass. However the values obtained in other countries may not be applied in Brazil ${ }^{1,5}$. Therefore, it is of scientific relevance to establish normal echocardiographic values in Brazilians citizens free of cardiovascular diseases and representing the ethnic and social composition of the communities.

In this study, we present echocardiographic data obtained in a representative sample of the adults living in the city of Porto Alegre.

\section{Methods}

An analytical, observational, population-based, cross-sectional study was performed. The process of proportional randomization was done in multiple stages and in conglomerates according to the data from the IBGE, relative to the 1991 census. The inclusion criteria were that participants had to be at least 18 years old and live in the selected residence. Among 1,219 individuals selected and interviewed in the domicile, a subsample was selected at random to undergo M-mode and two-dimensional echocardiography with color Doppler.

Initially, $10 \%$ of the study population was chosen, and 
an additional $10 \%$ was chosen to substitute for no shows, generating a total of 132 individuals. Of these, 75 individuals were evaluated, corresponding to $57 \%$ of the eligible study population. To complete the planned sample size and substitute for the no shows, 57 participants from the same sectors as the absentees were chosen at random, of which 39 showed up (68\%). The final sample size was 114 individuals. In both the first and second contact attempts, we proceeded, sequentially, from invitation by letter, phone call, and home visit, always paying for expenses. The analyses were restricted to the healthy participants, especially concerning pathologies of the cardiovascular system, excluded by history and physical examination. Additionally, valvular disease was excluded with the echocardiographic examination. No patient had a history of hypertension or use of cardiovascular drugs.

The sample size was calculated according to estimates of the accuracy of the measurements; therefore, we expected to study an adequate number of adults with reliable confidence intervals limited to the parameters of the survey population. Due to the absence of a solid background (studies of representative sampling of communities), we used the description of means and dispersion of measurements presented by studies that used systematic samples. Many of these studies describe the parameters and associations in samples of approximately 100 individuals, a number that was operationally feasible to meet in this survey.

M-mode and two-dimensional echocardiography with color Doppler was performed in the individuals in left lateral decubitus position, using na Aloka model 870 with transducer of $3.5 \mathrm{MHz}$ and with concomitant registration of an electrocardiographic lead. Three consecutive measurements were acquired for each echocardiographic parameter at the end of the expiration; and the mean of these three measurements was used as the final parameter. In all the patients, the long axis view of the heart was used. All the echocardiographic measurements were acquired according to the standards established by the American Society of Echocardiography ${ }^{6}$ and with the recommendations proposed by Devereux et al ${ }^{7}$.

Aorta, left atrium, interventricular septum, and posterior wall thickness, left ventricle in systole and diastole, and the right ventricle were evaluated. The beginning of the QRS complex (first deflection) was used as the area to obtain the measurements at the end of diastole and maximal incursion of the septal movement for the measurements of the systolic dimension of the left ventricle ${ }^{6}$. The cavity and wall thicknesses were measured at the level of the mitral chords ${ }^{6}$.

Left ventricular mass was calculated using the formula from Devereux et al $^{7}$ :

Left ventricular mass $(\mathrm{g})=0.80 \times[1.04\{(\mathrm{SED}+\mathrm{LVED}-$ + PWED $)^{3}$-(LVED $\left.\left.)^{3}\right\}\right]+0.6$ where SED is ventricular septum thickness in end diastole, LVED is end diastolic diameter of the left ventricle, and PWED is posterior wall thickness in diastole. We obtained the weight (kilograms), height (centimeters), body mass index (kilogram for square meter), and body surface area (calculated by the formula: weight ${ }^{0.425}$ $\mathrm{x}$ height $\left.{ }^{0.725} \mathrm{x} 72.84 / 10,000\right)$. Additional data, including alcohol consumption and socioeconomic level were collected through a standardized questionnaire.

The parameters in our study were described as means and estimates of distribution (standard deviation and confidence interval of $95 \%$ ). The analyses were done in the entire sample and stratified by gender. We present the $\mathrm{P}$ values, assuming statistical significance of $\mathrm{P}$ less than $5 \%$.

\section{Results}

The original sample had the distribution of sex and age and other representative demographic characteristics similar to the population of Porto Alegre.

A total of 100 out of 114 echocardiograms were done in participants free of cardiovascular diseases or other disorders clinically evident. Fifty-two $(52 \%)$ were female and $17(17 \%)$ declared themselves to be of African origin. Thirtynine (39\%) participants were smokers, with daily consumption of 14.6 \pm 13.9 cigarettes per day, $11(11 \%)$ were formersmokers, and $50(50 \%)$ had never smoked.

The characteristics of the individuals studied, stratified by sex, are presented in table I. Age, body mass index, and systolic and diastolic blood pressures were similar in men and women. Height, body surface area, weight, heart rate, alcohol consumption, and years of attendance in school were significantly higher in men. The heart rate was significantly higher in women.

To evaluate interobserver variability, another echocardiographer evaluated the measurements done in 11 patients. The means of the measurements done by both observers had a variability of less than $5 \%$ in all variables analyzed.

The echocardiographic measurements are presented, according to sex, in table II, including the values for left ventricular mass corrected by the body surface area and height. The dimensions of the aorta, left atrium, interventricular septum, left ventricle in systole and diastole, posterior wall, and left ventricular mass, corrected or not, were signi-

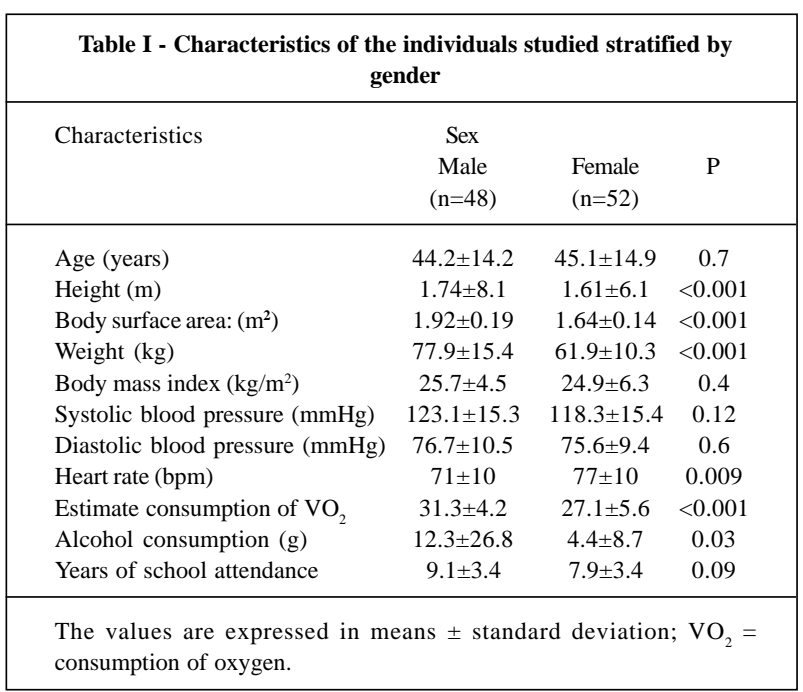


ficantly higher in men. The dimension of the right ventricle was similar in both sexes.

The 5 th and 95 th percentiles of the echocardiographic parameters are presented in table III.

The left ventricular mass of the white participants was $121.5 \pm 34.4 \mathrm{~g}$ and in black participants was $124.2 \pm 33.6 \mathrm{~g}$ $(\mathrm{P}=0.77)$. The ventricular mass corrected by height or body surface area was not statistically different between whites and blacks.

No statistically significant difference in left ventricular mass was found, absolute or corrected for height or body surface area, between current smokers, former-smokers, and participants who had never smoked.

\section{Discussion}

Studies with the purpose of defining normal parameters must describe the origin of the population and the sampling criteria. The absolute majority of the reference studies of normal values for echocardiographic measurements do not present this information. Reference values for the echocardiogram must originate from a representative population sample of the inhabitants free of cardiovascular disease or other illnesses clinically relevant to the parameter of interest. Many studies do not specify the origin of the studied population or use relatives of patients, employees, or individuals whose echocardiogram was interpreted as normal ${ }^{2-4,8,9}$. The sampling criteria of the present study differentiate it from most of the studies reported in the literature, because this survey offers an equivalent probability of the adult inhabitants of Porto Alegre to be selected.

The influence of the replacement by substitutes of some nominees who did not show up could not directly be determined. Considering that the number of absentees in the first invitation was relatively small and that the substitutes were recruited from the same geographical region and socioeconomic level, it can be assumed that a selection bias did not occur. The sample size allowed small dispersion of the echocardiographic parameters, as can be seen by the calculated $95 \%$ confidence intervals.

Difficulties in obtaining acceptable echocardiographic images occurred in 5\% of the participants, and they were excluded. Savage et al ${ }^{10}$ studied image quality in 6,148 men and women of in the Framingham study in the age group between 17-90 years old. The authors were able to obtain interpretable images in $80 \%$ of the cases, reaching $97 \%$ in younger patients. Obesity, decreased pulmonary capacity, and cardiovascular illness were associated with unacceptable echocardiograms, independent of the patient's age.

The analysis of interobserver variability between the two readers in the present study demonstrated that measurements did not significantly differ for left ventricular dimensions and function. The dispersion of the results is within the normal limits established in the literature ${ }^{11}$.

The differences between the echocardiographic parameters observed in this study and those presented in the literature are broad, for example, for septum and posteri-

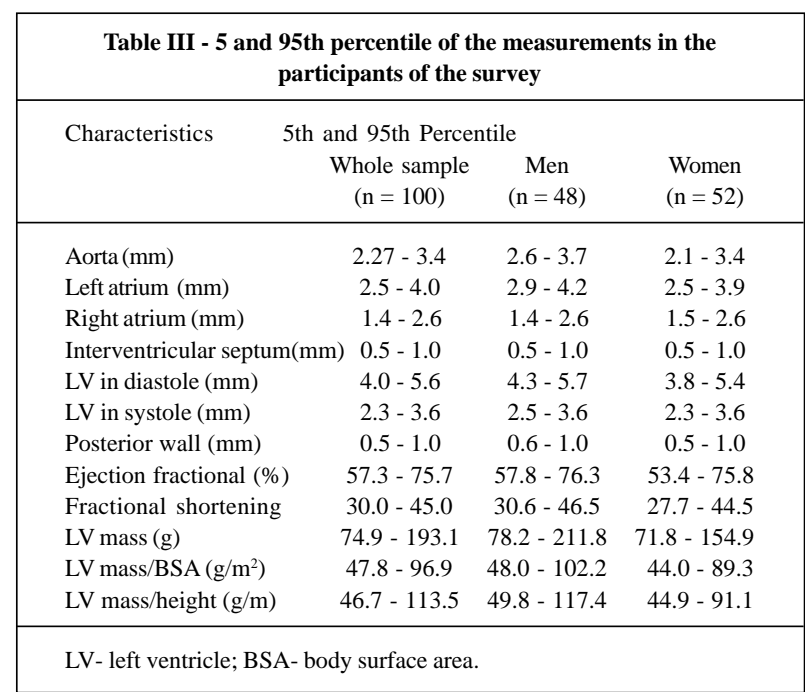

\begin{tabular}{|c|c|c|c|}
\hline Characteristics & $\begin{array}{c}\text { Sex } \\
\text { Male } \\
(\mathrm{n}=48)\end{array}$ & $\begin{array}{l}\text { Female } \\
(\mathrm{n}=52)\end{array}$ & $\mathrm{P}$ \\
\hline Aorta (mm) & $3.1 \pm 0.3(3.0-3.2)$ & $2.8 \pm 0.4(2.7-2.9)$ & $<0.001$ \\
\hline Left Atrium (mm) & $3.5 \pm 0.4(3.4-3.6)$ & $3.3 \pm 0.5(3.2-3.4)$ & 0.005 \\
\hline Right ventricle $(\mathrm{mm})$ & $2.1 \pm 0.4(2.0-2.2)$ & $2.0 \pm 0.4(1.9-2.1)$ & 0.6 \\
\hline Interventricular septum $(\mathrm{mm})$ & $0.8 \pm 0.2(0.7-0.9)$ & $0.71 \pm 0.1(0.68-0.74)$ & 0.006 \\
\hline $\mathrm{LV}$ in diastole $(\mathrm{mm})$ & $5.0 \pm 0.4(4.9-5.1)$ & $4.7 \pm 0.4(4.6-4.8)$ & $<0.001$ \\
\hline LV in systole (mm) & $3.2 \pm 0.3(3.1-3.3)$ & $2.9 \pm 0.4(2.8-3.0)$ & 0.01 \\
\hline Posterior wall $(\mathrm{mm})$ & $0.8 \pm 0.1(0.7-0.8)$ & $0.7 \pm 0.14(0.70-0.8)$ & 0.03 \\
\hline Ejection fraction (\%) & $66.4 \pm 5.5(64.8-68)$ & $65.8 \pm 6.1(64.1-67.5)$ & 0.6 \\
\hline Fractional shortening & $37.2 \pm 4.6(35.9-38.5)$ & $36.3 \pm 4.6(35-37.6)$ & 0.3 \\
\hline LV mass $(\mathrm{g})$ & $137.6 \pm 35.2(127.6-147.6)$ & $107 \pm 25.9(99.9-114.0)$ & $<0.001$ \\
\hline LV mass/BSA $\left(\mathrm{g} / \mathrm{m}^{2}\right)$ & $72.14 \pm 15.1(67.9-76.4)$ & $64.8 \pm 14.2(60.9-68.7)$ & 0.02 \\
\hline LV mass/height $(\mathrm{g} / \mathrm{m})$ & $79.1 \pm 19.2(73.7-84.5)$ & $66.7 \pm 15.7(62.4-71)$ & $<0.00$ \\
\hline
\end{tabular}


or wall thickness and aorta dimensions. Due to the measurements of the septum and the posterior wall, the left ventricular mass was also smaller than the reference values, corrected or not by body surface area or height. The normal limits are $1.0 \mathrm{~cm}$ for interventricular-septum and posterior wall defined by the 95 th percentile, versus $1.1 \mathrm{~cm}$ described in a textbook ${ }^{1}$. The means for these parameters found in this study also differed from those in the literature ${ }^{2,3,5,8,9,12}$. The differences have greater magnitude if the $95 \%$ confidence interval is used to define normality. The other echocardiographic measurements are within the normal values presented in the international literature ${ }^{2-5,8,9,12}$. At least one Brazilian study using a sample of 32 employees of a hospital, presented numbers similar to those in our study. Some of the differences may be due to different formulas applied to the estimation of left ventricular mass. The Penn method was used in that study, which excluded the endocardium surface of the parietal thickness and included this thickness in the cavity dimensions. The American Society of Echocardiography recommends that the measurements should use the most external echo (leading to leading edge) ${ }^{6}$. However, this produces a systematic superestimation of left ventricular mass when compared with that on autopsy. So it is necessary to correct the result by a factor derived from anatomical postmortem studies?

The same inference can explain part of the discrepancies between the values of left ventricular mass provided by different formulas in different echo equipment and the one found in our study. For example, in the equipment used in the present study, the Penn method for left ventricular mass calculation was used.

Despite the use of different formulas, unexplainable differences persist. The only consistent ones are criteria for randomization or from the study population, assuming that the examinations were done with technical expertise and according to international recomendations ${ }^{6}$. The first explanation is more reasonable because the ethnic constitution of the inhabitants of Porto Alegre is similar to that of European and North American countries. Anthropometric differences could only explain the absolute differences between the mentioned dimensions.

The smaller aortic dimension was also remarkable in relation to the values reported in the literature. Again, the different sampling criteria are the best available explanation for the discrepancy between the values obtained in our study and those presented in international textbooks.

In conclusion, the findings of this study permit to conclude that, in adults of the urban region of Porto Alegre, the means and the normal distribution conventionally accepted as the limits of normality for the dimensions of interventricular septum, posterior wall, and left ventricular mass are lower than those presented in the literature of reference; left ventricular mass is larger in men, corrected or not for anthropometric variables. The consequences of these findings for clinical practice are obvious as, for example, in the risk stratification of patients with hypertension.

\section{References}

1. Feigenbaum H. Echocardiography. $5^{\text {th }}$ Edition ed. Philadelphia: Lea \& Febiger; 1993.

2. Trivedi S, Gupta O, Jain A, et al. Left ventricular M-mode echocardiographic measurements of Indian population. J Assoc Physicians India 1993; 41: 14-16.

3. Knutsen K, Stugaard M, Michelsen S, et al. M-mode echocardiographic findings in apparently healthy, non-athletic Norwegians aged 20-70 years. Influence of age, sex, and body surface area. J Intern Med 1989; 225: 111-15.

4. MorcerfF, Thevenard R, Fuks J, et al. Ecocardiografia. Método e valores normais. Arq Bras Cardiol 1976;29:459-465. Arq Bras Cardiol 1976; 29: 459-65.

5. Shub C, Klein A, Zachariah P, et al. Determination of left ventricular mass by echocardiography in a normal population: effect of age and sex in addition to body size. Mayo Clin Proc 1994; 69: 205-11.

6. Sahn D, DeMaria A, Kisslo J, et al. The Committee on M-mode Standardization of the American Society of Echocardiography: recomendations regarding quantitation in M-mode echocardiography: results of a survey of echocardiographic methods. Circulation 1978; 58: 1072-83.
7. Devereux R, Alonso D, Lutas E, et al. Echocardiographic assesment of left ventricular hypertrophy: comparison with necropsy findings. Am J Cardiol 1986; 57 : 450-8.

8. Devereux R, Lutas E, et al. Standardization of M-mode echocardiographic left ventricular anatomic measurements. J Am Coll Cardiol 1984; 4: 1222-30.

9. Lauer M, Larson M, Levy D. Gender-specific reference M-mode values in adults: population-derived values with consideration of the impact of height. J Am Coll Cardiol 1995; 26: 1039-46.

10. Savage D, Garrison R, Kannel W, et al. Considerations in the use of echocardiography in epidemiology. The Framingham Study. Hypertension 1987; 9: II40-II4

11. Gardin J, Wong N, Bommer W, et al. Echocardiographic design of a multicenter investigation of free-living elderly subjects: the Cardiovascular Health Study. J Am Soc Echocardiogr 1992; 5: 63-72.

12. Marcus R, Krause L, Weder A, et al. Sex-specific determinants of increased left ventricular mass in the Tecumseh Blood Pressure Study. Circulation 1994; 90 : 928-936. 